\title{
Therapierelevante Daten zu gastrointestinalen Tumoren beim ASCO 2016
}

\section{Chemotherapie bei Patienten mit fortgeschrittenem hepatozellulärem Karzinom nicht wirksam}

In einer Phase-II-Studie hatte sich gezeigt, dass Patienten mit fortgeschrittenem hepatozellulärem Karzinom (HCC) signifikant länger lebten, wenn zur Standardtherapie Sorafenib noch Doxorubicin hinzugegeben wurde [1]. Eine beim ASCO Annual Meeting 2016 präsentierte Phase-III-Studie evaluierte die Wirksamkeit der Kombination aus Chemo- und zielgerichteter Therapie nun in einem größeren Patientenkollektiv [2].

\section{Primärer Endpunkt Gesamtüberleben}

Nicht vorbehandelte Patienten mit einem fortgeschrittenen Leberzellkarzinom und gutem Allgemeinzustand bekamen randomisiert entweder Doxorubicin $60 \mathrm{mg} / \mathrm{m}^{2}$ i.v. alle 21 Tage plus Sorafenib $400 \mathrm{mg}$ oral zweimal täglich oder eine Sorafenib-Monotherapie. Sorafenib wurde im Chemotherapie-Arm als Erhaltungstherapie weitergegeben. Bei einem 1,2× über normal erhöhten Bilirubinwert wurden die Dosen jeweils halbiert. Primärer Endpunkt war das Gesamtüberleben, sekundär wurde das progressionsfreie Überleben (PFS) ausgewertet. Ziel der Studie war es, eine Verlängerung des medianen Überlebens um 37\% mit der Kombination zu erreichen.

Insgesamt 356 Patienten mit einem medianen Alter von 62 Jahren wurden eingeschlossen. Die Teilnehmer waren überwiegend männlich (86\%) und nicht-asiatischer Herkunft (81,5\%). Bei mehr als der Hälfte der Patienten war die Erkrankung metastasiert $(57,5 \%)$.

\section{Weder Vorteil beim Gesamt- noch beim progressionsfreien Überleben}

Der primäre Endpunkt wurde nicht erreicht. Das mediane Gesamtüberleben betrug 8,9 Monate für die Kombination und 10,5 Monate für Sorafenib allein (Hazard Ratio $(\mathrm{HR})=1,06$; 95\%Konfidenzintervall-(CI) 0,8-1,4; $\mathrm{p}=0,24)$. Auch beim PFS zeigte sich mit 4,0 vs. 3,9 Monaten kein signifikanter Unterschied $(\mathrm{HR}=0,9 ; 95 \%$-CI $0,72-1,2 ; \mathrm{p}=0,98)$.

\section{Erhöhte Raten therapiebedingter Toxizitäten}

Hämatologische Nebenwirkungen $\geq$ Grad 3 traten erwartungsgemäß nur im Kombinationsarm auf. Aufgrund der Sorafenib-Therapie kam es gehäuft zu Bluthochdruck und Hand-Fuß-Syndrom $\geq$ Grad 3. Die Rate kardialer Toxizitäten $\geq$ Grad 3 betrug insgesamt 4,3\%. Es gab 12 als therapiebedingt eingestufte Todesfälle: $8 \mathrm{im}$ Prüfarm und 4 im Vergleichsarm. Ursachen im Kombinationsarm waren u. a. kardiale Komplikationen, Infektionen und Leberversagen.

\section{Fazit}

Die Addition von Doxorubicin zu Sorafenib führte zu einer deutlich höheren Toxizität. Weder das Gesamtüberleben noch das progressionsfreie Überleben wurden im Vergleich mit der Standardtherapie Sorafenib verbessert. Die CALGB 80802 Studie belegt damit, dass die Chemotherapie derzeit keinen Stellenwert bei der Behandlung des hepatozellulären Karzinoms hat.

\section{Adjuvante Kombinationschemotherapie mit Gemcitabin und Capecitabin beim Pankreaskarzinom wirksamer als Gemcitabin}

Das Studienprogramm der European Study Group for Pancreatic Cancer (ESPAC) hat das Ziel, die Chemotherapie bei Patienten mit einem resezierten Pankreaskarzinom weiterzuentwickeln. Standard in dieser Indikation ist eine Monotherapie mit Gemcitabin, seit in ESPAC-3 im Vergleich mit 5-FU ein vergleichbares Gesamtüberleben gezeigt werden konnte. Gemcitabin war dabei etwas besser verträglich [3]. Beim ASCO Annual Meeting 2016 wurden Ergebnisse der Studie ESPAC-4 als Late-Breaking-Abstract vorgestellt. Diese prüfte, ob die Zugabe von Capecitabin zu Gemcitabin das Überleben verbessert [4].

\section{Studiendesign}

Eingeschlossen wurden Patienten mit einem duktalen Adenokarzinom des Pankreas ohne Fernmetastasierung. Sie bekamen

\section{KARGER}

๑) 2016 S. Karger GmbH, Freiburg 
randomisiert innerhalb von 12 Wochen nach Operation entweder jeweils 6 Zyklen Gemcitabin $1000 \mathrm{mg} / \mathrm{m}^{2}$ i.v. an den Tagen 1, 8 und 15 oder Gemcitabin in Kombination mit Capecitabin oral $1660 \mathrm{mg} / \mathrm{m}^{2}$ pro Tag für 24 Wochen. Die Auswertung wurde unter anderem stratifiziert nach dem Resektionsstatus (R0/R1). Primärer Endpunkt war das Gesamtüberleben, zu den sekundären Endpunkten gehörten das 5-Jahresüberleben sowie die Verträglichkeit.

Insgesamt 732 Patienten wurden im Verhältnis 1:1 randomisiert. Das Alter lag im Median bei 65 Jahren, 57\% der Patienten waren männlich. Überwiegend hatten die Teilnehmer einen guten WHO-Performance-Status (0: 42\%, 1: 55\%). Die maximale Tumorgröße lag im Median bei $30 \mathrm{~mm}$. Bei 40\% der Patienten wurde eine R0- und bei $60 \%$ eine R1-Resektion erreicht. $80 \%$ der Tumoren erwiesen sich als nodal positiv. In 50\% der Fälle handelte es sich um ein moderat differenziertes, bei $39 \%$ um ein schlecht differenziertes und bei 9\% um ein hochdifferenziertes Karzinom.

\section{Signifikante Verbesserung des Überlebens um 2,5 Monate}

Die Kombinationschemotherapie war der Gemcitabin-Monotherapie bezüglich des Gesamtüberlebens signifikant überlegen. Die Patienten im Capecitabin-Arm lebten im Median 28 Monate im Vergleich zu 25,5 Monaten mit Gemcitabin allein ( $\mathrm{HR}=0,82$; $95 \%$-CI $0,68-0,98 ; \mathrm{p}=0,032$ ). Nach fünf Jahren waren noch $28,8 \%$ der Patienten im Kombinationsarm und 16,3\% in der Kontrollgruppe am Leben. Die Stratifikation nach dem Resektionsstatus ergab keinen Unterschied zwischen R0 und R1.

Insgesamt 25\% der Patienten berichteten 305 therapiebedingte Nebenwirkungen von Grad 3/4, 24\% davon traten im Kombinationsarm auf und 26\% mit Gemcitabin allein. $\mathrm{Zu}$ den unerwünschten Ereignissen, die unter der kombinierten Therapie signifikant häufiger auftraten als unter Gemcitabin, gehörten Neutropenie, Hand-Fuß-Syndrom und Diarrhoe.

\section{Fazit}

Die adjuvante Kombinationschemotherapie aus Gemcitabin und Capecitabin zeigte einen statistisch signifikanten Vorteil gegenüber einer Gemcitabin-Monotherapie bei Patienten mit Pankreaskarzinom. Die Ergebnisse der ESPAC-4-Studie legen nahe, dass eine Intensivierung der adjuvanten Chemotherapie beim resezierten Pankreaskarzinom zu einer Verbesserung der Prognose beiträgt. Ob Gemcitabin + Capecitabin letztlich ein besonders empfehlenswertes Behandlungsschema sein wird, sollte abgewartet werden. Adjuvante Therapiestudien zu Gemcitabin + nab-Paclitaxel oder FOLFIRINOX im Vergleich zu Gemcitabin mono stehen ebenfalls kurz vor dem Abschluss. Bis auf weiteres kann Gemcitabin mono für 6 Monate noch als eine adjuvante Standardtherapie des resezierten Pankreaskarzinoms angesehen werden. Änderungen dieses Standards stehen bevor.

\section{Kein Vorteil für postoperative Chemoradiotherapie bei Patienten mit resektablem Magenkarzinom vs. Chemotherapie}

Die wichtigste potenziell kurative Therapie des Magenkarzinoms und von Tumoren des gastroösophagealen Übergangs (GEJ) ist die Operation, gefolgt von einer postoperativen Chemotherapie [5]. Da die Erkrankung bei Erstdiagnose häufig jedoch bereits fortgeschritten ist, beträgt die Langzeitüberlebensrate nach 15 Jahren nur etwa 25\% [6]. Die Phase-III-Studie CRITICS untersuchte, ob eine Radiochemotherapie nach neoadjuvanter Chemotherapie und adäquater Resektion zu einem besseren Überleben führt als die postoperative Chemotherapie [7].

Eingeschlossen wurden Patienten mit einem operablen Magenkarzinom oder GEJ-Tumor im Stadium IB-IVa und einem WHOPerformance-Status 0-1. Die neoadjuvante Chemotherapie in beiden Armen bestand aus 3 Zyklen Epirubicin, Cisplatin oder Oxaliplatin und Capecitabin. Nach der Operation wurde die Chemotherapie entweder mit weiteren 3 Zyklen fortgesetzt oder mit einer Strahlentherapie intensiviert (Chemoradiotherapie). Primärer Endpunkt war das Gesamtüberleben, sekundäre Endpunkte das progressionsfreie Überleben (PFS) und die Verträglichkeit.

\section{Nur etwa die Hälfte der Patienten beendete die Therapie}

Die randomisierte, multizentrische Studie schloss 788 Patienten ein, die im Verhältnis 1:1 randomisiert wurden. Im Median waren sie 62 Jahre alt, überwiegend handelte es sich um Männer (67\%). Fast ein Fünftel der Teilnehmer hatte einen GEJ-Tumor (17\%). 85\% der Patienten erhielten alle 3 Zyklen der neoadjuvanten Chemotherapie. Bei fast der Hälfte erfolgte eine D1-Resektion, die Rate an pt3/pT4-Tumoren lag bei 44\%. Im Chemotherapie-Arm beendeten $47 \%$ und im Chemoradiotherapie-Arm 52\% die Behandlung per Protokoll. Zu den Gründen für das Nicht-Antreten der postoperativen Behandlung gehörten Ablehnung durch den Patienten, Krankheitsprogress und die Toxizität der neoadjuvanten Chemotherapie.

\section{Gesamtüberleben vergleichbar}

Beim Vergleich des Gesamtüberlebens zwischen Chemotherapie- und Chemoradiotherapie-Patienten zeigte sich kein signifikanter Unterschied (3,5 vs. 3,3 Jahre; $\mathrm{p}=0,99)$. Dies war auch bei den Überlebensraten nach 5-Jahren noch der Fall (40,8 vs. 40,9\%). Ebenso konnte das PFS durch die intensivierte Therapie im Vergleich zur Chemotherapie nicht verbessert werden (2,3 vs. 2,5 Monate; $\mathrm{p}=0,99$ ).

Bei den Patienten, die die postoperative Therapie abgeschlossen hatten $(\mathrm{n}=486)$ waren die häufigsten unerwünschten Ereignisse $\geq$ Grad 3 hämatologischer und gastrointestinaler Natur. Im Chemotherapie-Arm traten signifikant häufiger Neutropenien auf als im experimentellen Arm (34 vs. 4\%; p < 0,001). 
Fazit

Es zeigte sich kein signifikanter Unterschied beim Gesamtüberleben zwischen Chemotherapie und Chemoradiotherapie im postoperativen Setting. Damit legen die Ergebnisse der CRITICS Studie nahe, dass es nach qualitätsgesicherter Chirurgie im perioperativen Chemotherapiekonzept, welches der empfohlene Standard für Patienten in Europa ist, keinen Stellenwert für eine zusätzliche adjuvante Radiotherapie gibt. Subgruppenanalysen mit Bezug auf spezifische Untergruppen (z.B. intestinaler Tumortyp, R1-Status oder ypN+ Status) werden in den nächsten Monaten präsentiert werden. Schon jetzt lässt sich aus den negativen Ergebnissen der CRITICS Studie allerdings ableiten, dass ein relevanter Benefit für eine größere Subgruppe von Patienten sehr unwahrscheinlich ist. Hinzu kommt die unbefriedigende Verträglichkeit und Durchführbarkeit einer adjuvant-postoperativ verabreichten Therapie. Eine Intensivierung der postoperativen Therapie im perioperativen Behandlungskonzept ist nutzlos. Zukünftige und laufende Behandlungskonzepte fokussieren auf die neoadjuvant-präoperative Behandlungsphase.

Dr. Jeannette Haeri in Zusammenarbeit mit Prof. Florian Lordick, Leipzig

\section{Literatur}

Abou-Alfa GK, et al.: Doxorubicin plus sorafenib vs doxorubicin alone in patients with advanced hepatocellular carcinoma: a randomized trial. JAMA 2010 ;304:2154-2160.

2 Abou-Alfa GK, et al.: Phase III randomized study of sorafenib plus doxorubicin versus sorafenib in patients with advanced hepatocellular carcinoma (HCC): CALGB 80802 (Alliance). J Clin Oncol 2016;34 (suppl; abstr 4003); oral presentation.

3 Neoptolemos JP, et al.: Adjuvant chemotherapy with fluorouracil plus folinic acid vs gemcitabine following pancreatic cancer resection: a randomized controlled trial. JAMA 2010;304:1073-1081.
4 Neoptolemos JP, et al.: ESPAC-4: A multicenter, international, open-label randomized controlled phase III trial of adjuvant combination chemotherapy of gemcitabine (GEM) and capecitabine (CAP) versus monotherapy gemcitabine in patients with resected pancreatic ductal adenocarcinoma. J Clin Oncol 2016;34 (suppl; abstr LBA4006); oral presentation.

5 S3-Leitlinie 'Magenkarzinom'. Z Gastroenterol 2011; 49:461-531.
6 Songun I, et al.: Surgical treatment of gastric cancer: 15-year follow-up results of the randomised nationwide Dutch D1D2 trial. Lancet Oncol 2010;11:439-449.

7 Verheij M, et al.: A multicenter randomized phase III trial of neo-adjuvant chemotherapy followed by surgery and chemotherapy or by surgery and chemoradiotherapy in resectable gastric cancer: first results from the CRITICS study. J Clin Oncol 2016;34 (suppl; abstr 4000); oral presentation. 